\title{
Haemolytic-uraemic syndrome presenting as an acute abdomen
}

\author{
P.M. Lamont* and A.I.G. Davidson \\ Department of Surgery, Perth Royal Infirmary, Perth PHI INX, UK.
}

\begin{abstract}
Summary: Haemolytic-uraemic syndrome (HUS) may present with symptoms and signs suggestive of an acute surgical condition of the abdomen. The patient described was admitted with clinical features sufficient to warrant operation but later proved to have HUS.
\end{abstract}

\section{Introduction}

Haemolytic-uraemic syndrome (HUS) is a disease of childhood characterized by haemolytic anaemia, thrombocytopenia and anuria. Prodromal gastrointestinal symptoms may occur, and these may occasionally be of sufficient severity that they mimic an acute abdominal emergency. We describe a case of HUS presenting with prodromal symptoms and signs which were initially attributed to intestinal intussusception requiring emergency surgery.

\section{Case report}

An 8 year old boy was admitted as an emergency with a 36-hour history of colicky lower abdominal pain, vomiting and diarrhoea, accompanied by the passage of fresh blood per rectum. Abdominal examination revealed tenderness, maximal in the left iliac fossa, with associated guarding and fullness in that area. Rectal examination confirmed blood on the examining finger. Abdominal X-rays showed gaseous distension of the caecum, ascending and transverse colon. Haemoglobin was $15.0 \mathrm{~g} / \mathrm{dl}$, white cell count was $15 \times 10^{9} / 1$, and plasma urea and electrolytes were within normal limits.

After a period of observation, the pain worsened and the child became more distressed.

At laparotomy there was some free blood-stained fluid in the peritoneal cavity. The appendix was normal. The sigmoid colon was oedematous with no obvious sign of ischaemia and normal pulsation in the mesocolic vessels. The appearances were attributed to spontaneous resolution of a colo-colic intussusception

Correspondence: A.I.G. Davidson, Ph.D., Ch.M., F.R.C.S. Ed.

*Present address: Westminster Hospital, London, SW1 UK. Accepted: 16 April 1987 and the abdomen was closed.

The initial postoperative recovery was uneventful but on the fifth day the patient vomited bile-stained fluid. The abdomen was soft and not distended. Within a further 24 hours the patient was oliguric with microscopic haematuria and proteinuria. The blood pressure had risen from $100 / 70$ to $120 / 90 \mathrm{~mm} \mathrm{Hg}$. Plasma electrolytes were normal but urea had risen to $31 \mathrm{mmol} / \mathrm{l}$. Haemoglobin had fallen to $9.0 \mathrm{~g} / \mathrm{dl}$ and a blood film showed $6 \%$ reticulocytes with many fragmented and deformed red blood cells or schistocytes, the appearances being diagnostic of HUS. Platelet count was $75 \times 10^{9} / 1$ and coagulation screen was normal apart from an increase in fibrin degradation products. Direct Coombs' test was negative and serum immunoglobulins and complement were normal. Urine culture was sterile.

By the eighth postoperative day the patient was anuric, had suffered a grand mal convulsion and was noted to have multiple skin petechiae. He was treated with blood transfusions, peritoneal dialysis, nutritional support and anticonvulsant and antihypertensive drugs. Seven weeks of continuous ambulatory peritoneal dialysis were required until renal function returned to normal. Dialysis was complicated by two episodes of candidal peritonitis.

The patient was discharged home 3 months after his original admission with apparently normal renal function, blood pressure and plasma urea and electrolyte levels. A year later he is well, on no treatment and with no obvious sequelae to his illness.

\section{Discussion}

Haemolytic-uraemic syndrome can present with prodromal gastrointestinal symptoms which may include abdominal pain, diarrhoea and vomiting.

(C) The Fellowship of Postgraduate Medicine, 1987 
These symptoms may precede the onset of anaemia and anuria by up to 3 weeks. ${ }^{1}$ The condition occurs typically in infants and young children and the features may mimic appendicitis. ${ }^{2}$ The presence of rectal bleeding can suggest a diagnosis of ulcerative colitis, ${ }^{3}$ and the abdominal colic, when it occurs in association with rectal bleeding, may be severe enough to suggest intussusception. In a large series reported by Gianantonio et al., twelve patients presented with features suggestive of intussusception. ${ }^{4} \mathrm{~A}$ fatal case has also been described following laparotomy for intussusception. ${ }^{5} \mathrm{~A}$ further two cases of spontaneously resolving intussusception have been reported as a complication of HUS. ${ }^{6}$

The aetiology of HUS has not yet been fully elucidated. Both sporadic and epidemic forms of the syndrome may occur and a variety of initiating agents have been implicated. ${ }^{7}$ The sporadic form has been noted in siblings, with periods in excess of one year between the onset in each sibling. An inherited predisposition to the syndrome has therefore been suggested, with transmission as an autosomal recessive trait. ${ }^{8}$ Epidemic outbreaks of the syndrome suggest the presence of an infectious initiating agent and a variety of organisms have been implicated, including shigella ${ }^{9}$ and verotoxin producing Escherichia coli. ${ }^{10,11}$ While no one particular organism has been isolated in every case, such heterogeneity may help to explain the differences in severity and prognosis of the syndrome which have been observed in different series. The epidemic form tends to occur in younger children with a diarrhoeal prodrome and a good prognosis, ${ }^{12}$ although the prognosis for recovery of renal function appears to vary in different epidemics. ${ }^{11}$ The sporadic form occurs in older children with less marked prodromal symptoms but a worse prognosis for renal function. $^{12}$

The pathogenesis of HUS is also unclear, although microvascular thrombotic occlusion with subsequent ischaemia accounts for many of the clinical manifestations. ${ }^{7}$ The initial stimulus for thrombosis may be related to endothelial damage by endotoxins or circulating antigen-antibody complexes. ${ }^{1,9}$ More recent interest has focussed on prostacyclin, a potent inhibitor of platelet aggregation. Both prostacyclin deficiency and the presence of a prostacyclin inhibitor have been reported in the plasma of HUS patients. ${ }^{13,14}$ Successful resolution of HUS has occurred after

\section{References}

1. Lieberman, E. Hemolytic-uremic syndrome. $J$ Pediatr 1972, 80: $1-16$.

2. Edmonson, M.B. \& Chesney, R.W. Hemolytic-uremic syndrome confused with acute appendicitis. Arch Surg 1978, 113: 754-755. infusions of either fresh plasma or prostacyclin, $\frac{\varrho}{3}$ although a response is not seen in every case. ${ }^{15,16} \stackrel{\mathbb{Q}}{\complement}$ Anticoagulation with heparin has also had variable $C$. success and is now seldom used as it does not appear to $\vec{F}$ alter the course of the disease to any significant extent. ${ }^{11}$

Microvascular occlusion and subsequent cortical $\frac{\bar{\sigma}}{\overline{\bar{V}}}$ necrosis explain the renal manifestations of HUS, with $\frac{\sigma}{7}$ irreversible damage and chronic renal failure develop- $\stackrel{\Phi}{\varrho}$

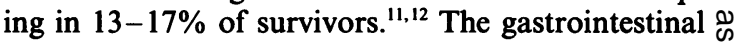
symptoms are often attributed to ischaemic colitis $\overrightarrow{0}$ from small vessel thrombosis. The ischaemia is usually self-limiting but may progress to colonic stricture, $\vec{\omega}$ perforation, toxic megacolon or frank infarction. ${ }^{17} \stackrel{\mathrm{\rho}}{\circ}$ Radiological features on barium enema include $\stackrel{\circ}{\circ}$ colonic mucosal oedema, 'thumb-printing', marginal 3 . serration and scattered filling defects, and may suggest $\delta$ acute ulcerative colitis. ${ }^{18}$ The thickened, oedematous $-v$ sigmoid colon seen at laparotomy in our patient was $\vec{c}$ consistent with the presence of ischaemic colitis, although the severity of the abdominal symptoms and $v$ signs precluded barium studies prior to operation.

Other extrarenal lesions described in HUS include $\rightarrow$ insulin-dependent diabetes, possibly arising secondary $\mathcal{D}_{\mathbb{D}}$ to microthrombosis of the pancreatic vasculature, ${ }^{19}$ cardiomyopathy" and neurological abnormalities $\stackrel{\mathbb{D}}{3}$ which may be a prominent feature. Convulsions, $\frac{\nabla}{\square}$ stupor, coma, decerebrate rigidity and hemiparesह have been described and while some cases are associated with microthrombosis of the cerebral vas. culature, ${ }^{19}$ microthrombi are not invariably present.${ }^{29}$ The commonest neurological manifestation would appear to be seizures related to hyponatraemia, ${ }^{11,20}$ which often occur near the time of admission and may be exacerbated by inappropriate fluid administration. Hypertension is also a contributing factor and may persist in surviving patients. These extrarenal manifestations all contribute to the significant mortality associated with HUS of between $9-16 \%$ in UK series. ${ }^{11} 12$ The subsequent risks of hypertension and chronic renal failure in survivors demands long term follow-up.

Haemolytic-uraemic syndrome may present to the surgeon mimicking appendicitis, ulcerative colitis or, as in the present case, intussusception. The onset of unexplained oliguria and anaemia in a child with prior gastro-intestinal symptoms should raise the possibility of haemolytic-uraemic syndrome.

3. Berman, W, Jr. The hemolytic-uremic syndrome; initial clinical presentation mimicking ulcerative colitis. $J$ Pediatr 1972, 81: 275-278.

4. Gianantonio, C.A., Vitacco, M., Mendilaharzu, F., $\mathbb{D}$ Gallo, G.E. \& Sojo, E.T. The hemolytic-uremic syn- 
drome. Nephron 1973, 11: 174-192.

5. Maki, S., Miyata, H. \& Uda, H. Hemolytic-uremic syndrome: a fatal case after operation for intussusception as a complication of upper respiratory infection. Med $J$ Osaka Univ 1968, 19: 157-173.

6. Sorrenti, L.Y. \& Lewy, P.R. The hemolytic-uremic syndrome. Am J Dis Child 1978, 132: 59-62.

7. Levin, M. \& Barratt, T.M. Haemolytic uraemic syndrome. Arch Dis Child 1984, 59: 397-400.

8. Kaplan, B.S., Chesney, R.W. \& Drummond, K.N. Haemolytic uraemic syndrome in families. $N$ Engl J Med 1975, 292: 1090-1093.

9. Koster, F., Levin, J., Walker, L. et al. Haemolyticuraemic syndrome after shigellosis: relation to endotoxaemia and circulating immune complexes. $N$ Engl J Med 1978, 298: 927-933.

10. Karmali, M.A., Steele, B.T., Petric, M. \& Lim, C. Sporadic cases of haemolytic-uraemic syndrome associated with faecal cytotoxin and cytotoxin producing Eschericia coli in stools. Lancet 1983, i: 619-620.

11. Taylor, C.M., White, R.H.R., Winterborn, M.H. \& Rowe, B. Haemolytic-uraemic syndrome: clinical experience of an outbreak in the West Midlands. $\mathrm{Br}$ Med $J$ 1986, 292: 1513-1516.

12. Trompeter, R.S., Schwartz, R., Chantler, C. et al. Haemolytic-uraemic syndrome: an analysis of prognostic features. Arch Dis Child 1983, 58: 101-105.

13. Remuzzi, G., Misiani, R., Marchesi, D. et al.
Haemolytic-uraemic syndrome: deficiency of plasma factor(s) regulating prostacyclin activity? Lancet 1978, ii: $871-872$.

14. Levin, M., Elkon, K.B. \& Nokes, T.J. Inhibitor of prostacyclin production in sporadic haemolytic uraemic syndrome. Arch Dis Child 1983, 58: 703-708.

15. Misiani, R., Appiani, A.C., Edefonti, A. et al. Haemolytic uraemic syndrome: therapeutic effect of plasma infusion. Br Med J 1982, 285: 1304-1306.

16. Defreyn, G., Proesmans, W., Machin, S.J., Lemmens, F. \& Vermylen, J. Abnormal prostacyclin metabolism in the hemolytic uremic syndrome: equivocal effects of prostacyclin infusions. Clin Nephrol 1982, 18: 43-49.

17. Stiegmann, G.V. \& Lilly, J.R. Surgical lesions of the colon in the hemolytic-uremic syndrome. Surgery 1979 , 85: 357-359.

18. Peterson, R.B., Meseroll, W.P., Shrago, G.G. \& Gooding, C.A. Radiologic features associated with the hemolytic-uremic syndrome. Radiology 1976, 118: 667671.

19. Upadhyaya, K., Barwick, K., Fishaut, M., Kashgarian, M. \& Siegel, J. The importance of nonrenal involvement in hemolytic uremic syndrome. Pediatrics 1980, 65: 115-120.

20. Bale, J.F., Brasher, C. \& Siegler, R.L. CNS manifestations of the hemolytic-uremic syndrome. Am J Dis Child 1980, 134: 869-872. 